\title{
Key Point and Block based Feature Matching for Effective Image Forgery Detection
}

\author{
Durga Jetthy $^{1}$, Purva Gubbawar ${ }^{1}$, Aditya Kumar Gupta ${ }^{1}$, Mrs. S.A.Panwar ${ }^{1}$ \\ E \& TC Department, Smt.Kashibai Navale College of Engineering, Pune ${ }^{1}$
}

\begin{abstract}
An effective method for the detection of forgery in images is proposed. In recent years, digital images are in use in many applications and for multiple purposes. They also play an crucial role in the storage and transfer of visual information, especially the secret ones. With this widespread utilization of digital images, in sum to the increasing number of tools and software of any digital image editing, now a days it is easy to manipulate and change the actual information of the image. Therefore, it has become mandatory to check the genuineness and the coalition of the image by using modern and digital techniques, which contribute to analysis and perception of the images content, and then make sure of their integrity. There are many types of image forgery, the most important and popular type is called copy move forgery, which uses the same image in the process of forgery. This type of forgery is used for one of two things, first to hide an object or scene by copying the area of the image and pasting it on another area of the same image. The second is the repetition of object or scene with change in some qualities "such as size and degree" by copying this object and pasting it on another area of the same image. The copy paste forgery in the input images can be identified with the help of segmentation, feature extraction and feature matching process. The input images were initially over segmented with the help of the SLIC algorithm. The features were extracted from each blocks based on Scale Invariant feature extraction algorithm (SIFT). From the extracted features the forgery is detected based on block matching and labeled feature point matching. In block matching process the distance between the divided images regions were identified. From the identified similarity between the features the copy move regions were identified in the image. The performance of the process is measured with the help of precision, recall and F-measure of the input image.
\end{abstract}

Keywords: feature point, feature block, SIFT, SLIC, etc.

\section{INTRODUCTION}

In this era, Digital Image Forgery has been increasingly easy to perform, so the reliability of the image is thus becoming an important issue to be focused on. By using the tool such as Adobe Photoshop, GIMP, Coral Paint fake images can be created.

The basic method for copy move forgery detection are block based and key point based. In DCT (discrete cosine transform) algorithm the input image is taken. Then DCT values extracted from the images were reduced based on optimization process based Principal Component Analysis (PCA). A discrete cosine transform (DCT) expresses a finite sequence of data points in terms of a sum of cosine functions oscillating at different frequencies.

The use of cosine rather than sine functions is critical for compression, since it turns out (as described below) that fewer cosine functions are needed to approximate a typical signal, whereas for differential equations the cosines express a particular choice of boundary conditions. DCTs are operating on real data with even symmetry.

Transformations like Discrete wavelet Transform (DWT), Singular Value Decomposition (SVD) and Fourier-Mellin Transform (FMT) were employed for the identification of the copy move regions in the images. The block features were measured based on Zernike moments, Average Gray value and entropy were extracted as block features for the identification of the copy move regions in the images. The second is the DWT. The DWT is any wavelet transform for which the wavelet are discretely sampled. It is basically used for getting low frequency signals and it decomposes a signal into a set of basis function. These basis functions are called as the wavelets.

There are many types of wavelets such as Haar, Mexican hat, battle L-Marrie. The singular value decomposition (SVD) is a factorization of a real or complex matrix. It is the generalization of the eigen decomposition of a positive semi definite normal matrix (for example, a symmetric matrix with positive eigenvalues) to any $\mathrm{m} \times \mathrm{n}$ matrix via an extension of polar decomposition.

Zernike moments used in this study are a class of such orthogonal moments. The reason for selecting them from among the other orthogonal moments is that they possess a useful rotation invariance property.

Another main property of Zernike moments is the ease of image reconstruction. The orthogonality property enables one to separate out the individual contribution of each order moment (its information content) to the reconstruction process. Simple addition of these individual contributions generates the reconstructed image. 
In key point based method the SIFT and SURF algorithms are used. In SIFT (scale invariant feature transform) algorithm the key points are first extracted from a set of reference image and it is stored in a database.

An object recognised in a new image by individually comparing each feature from new image to the database and finding each matching feature based on Euclidian distance.

In SURF (speeded up robust features) algorithm is a local feature detector and descriptor. It can be used for tasks such as object recognition and 3D reconstruction.

\section{METHODOLOGY}

\section{A. COMPARISON BETWEEN DIFFERENT TYPES OF IMAGE FORGERY}

\section{Copy move:}

This is the most common kind of image tampering technique used, where one covers a part of the image in order to add or remove information. Textured regions are used as parts for copy-move[15,16] forgery, because textured areas have similar color, dynamic range, noise variation properties to that of the image.

This project focuses on copy-move forgery, that can be done very easily by using the tools such as Cloning in Photoshop. In this type of tampering, portions of an image are copied and pasted in other portions of the same image to conceal a person or object in the scene.

\section{Image Splicing}

A pasting of a image by sticking together photographic images. Splicing $[15,16]$ is a technique which involves composite of two or more images to create a new fake image.

\section{Resize}

Resize [17] operation performs a geometric transformation which can be used to shrink or enlarge the size of an image or part of an image

\section{Cropping}

It is a technique to cut-off borders of an image. Generally this kind of operation is used to remove border information.

\section{Noise and Blurring}

Tampering images with operations like image splicing, scaling, rotating can sometimes be clear to a viewer in the form of improper edges, aliasing defects and tone variations.

These obvious traces of tampering can be made imperceptible by applying small amount of noise or blur [18] operations in the portions where the tampering defects are visible.

\section{B. FLOW DIAGRAM:}

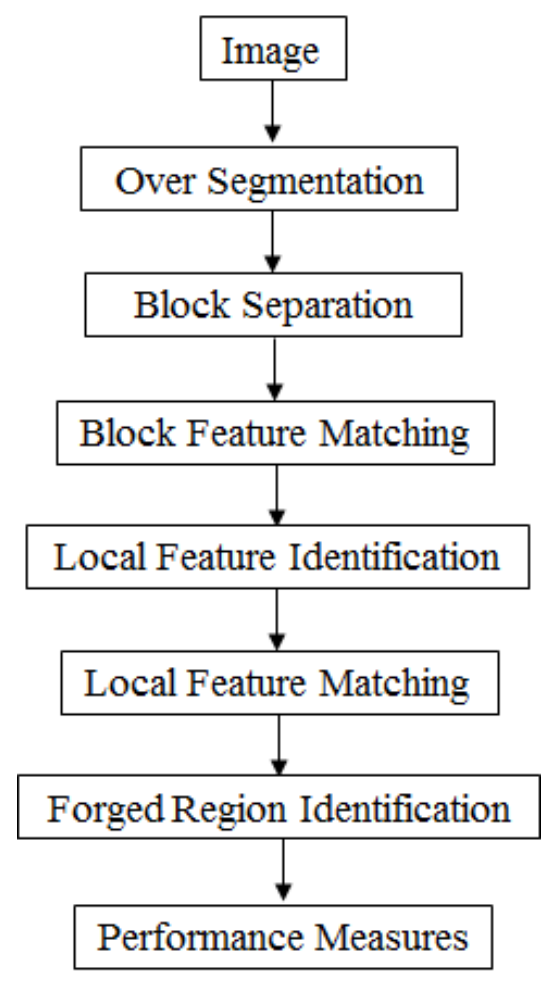

\section{PROPOSED SYSTEM}

The process of forgery detection in images is employed based on the feature point based and block based matching process. The block size of the images were calculated based on the input image's DCT transformation. The images were over segmented with the help of Simple Linear Iterative Clustering (SLIC) algorithm. The SLIC algorithm segments the images based on the block size determined using DCT transformation. SLIC uses the same compactness parameter (chosen by user) for all super pixels in the image. If the image is smooth in certain regions but highly textured in others, SLIC produces smooth regular-sized super pixels in the smooth regions and highly irregular super pixels in the textured regions. $\mathrm{K}$ regularly spaced cluster centers were sampled and they were moved to seed locations corresponding to the lowest gradient position in a $3 \times 3$ neighborhood. This is done to avoid placing them at an edge and to reduce the chances of choosing a noisy pixel. Each pixel in the image is associated with the nearest cluster center whose search area overlaps this pixel. After all the pixels are associated with the nearest cluster center, a new center is computed as the average lab xy vector of all the pixels belonging to the cluster. We then iteratively repeat the process of associating pixels with the nearest cluster center and recomputing the cluster center until convergence. At the end of this process, a few stray labels may remain, that is, a few pixels in the vicinity of a larger segment having the same label but not connected to it. The block features (BF) were extracted based on Scale Invariant Feature Transformation (SIFT) process. The derivative of the 
images is calculated. The calculated values gives the 3 changes in the color and the gray scale values of the image which indicates the informations in the image. The 4 . laplacian function calculates the edges in the images based on the derivative values. The values are then arranged in order in a matrix format. The values in a particular circle region is first chosen. The values in the chosen region were dilated. In the dilation process the values are compared and the values that have the lowest values are combined. Then the values that having the minimum values are then removed. The resulting points are saved as the HRL points. The Obtained HRL points are then used along with the image inorder to find the main orientation points in the image. The Edges of the histogram points were calculated and for the calculated values derivative values are calculated so that the obtained points were more optimized to get the exact edge values. The obtained values are shifted based on the particular angles to optimize the obtained values more clearly.

Then the optimal values are selected from the given set of values based on the gradient calculation and max value and max intensity value calculation. Finally the calculated values are padded with the image pixels and their corresponding ids were obtained and then the values are saved as the main orientation points. It is an algorithm in computer vision to detect and describe local features in images. For any object in an image, interesting points on the object can be extracted to provide a "feature description" of the object. This description, extracted from a training image, can then be used to identify the object when attempting to locate the object in a test image containing many other objects. To perform reliable recognition, it is important that the features extracted from the training image be detectable even under changes in image scale, noise and illumination. Such points usually lie on high-contrast regions of the image, such as object edges. Another important characteristic of these features is that the relative positions between them in the original scene shouldn't change from one image to another. First the extrema is calculated for the Scale-Space.

Then the key points are localized. The nearby points are interpolated. The Low contrast key points and the edge responses are eliminated. From the block features extracted labeled feature points (LFP) were calculated. The LFP were matched inorder to identify the forges regions in the images. The performance of the process is measured with the help of performance metrics like Precision, Recall value estimated.

\section{Drawbacks of block based method:}

1. The process were based on block matching process and hence when the image size increases the time complexity and algorithm complexity increases and also the features extracted based on the block based approaches.

2. The block based methods cannot exactly identify the copy move areas.
The shape regions cannot be exactly identified based on the block based approaches.

4. The performance of the process measured based on performance metrics indicates that the approaches needs improvement further.

\section{SLIC segmentation algorithm}

\section{i. SLIC SEGMENTATION}

The images were over segmented with the help of Simple Linear Iterative Clustering (SLIC) algorithm. The SLIC algorithm segments the images based on the block size determined using DCT transformation. SLIC uses the same compactness parameter (chosen by user) for all super pixels in the image. If the image is smooth in certain regions but highly textured in others, SLIC produces smooth regular-sized super pixels in the smooth regions and highly irregular super pixels in the textured regions.

So, it become tricky choosing the right parameter for each image. $\mathrm{K}$ regularly spaced cluster centers were sampled and they were moved to seed locations corresponding to the lowest gradient position in a $3 \times 3$ neighborhood. This is done to avoid placing them at an edge and to reduce the chances of choosing a noisy pixel. Each pixel in the image is associated with the nearest cluster center whose search area overlaps this pixel.

After all the pixels are associated with the nearest cluster center, a new center is computed as the average lab xy vector of all the pixels belonging to the cluster. We then iteratively repeat the process of associating pixels with the nearest cluster center and recomputing the cluster center until convergence. At the end of this process, a few stray labels may remain, that is, a few pixels in the vicinity of a larger segment having the same label but not connected to it. While it is rare, this may arise despite the spatial proximity measure since our clustering does not explicitly enforce connectivity. SLIC is a simple and efficient method to decompose an image in visually homogeneous regions. It is based on a spatially localized version of $\mathrm{k}$ means clustering.

\section{Advantages of SLIC algorithm:}

1. The performance metrics measured indicates that the proposed approach is more efficient as compared to the existing approaches.

2. The process produces non overlapping irregular blocks based on SLIC algorithm which produces more effective identification of the forged regions in the images.

3. The process can able to identify copy move regions that are transformed. The objects that were in irregular shapes can also be identified.

4. The process of combinations of the block based approaches and the feature point based approach reduces the time consumption and the algorithm complexity of the process. 


\section{DISCUSSION AND CONCLUSION}

\section{A. APPLICATION}

1. To detect if forgery has occurred.

2. It serves the purpose to catch the tampered or Fraudulent image.

3. This feature can help reduce online forgery or forgery done by powerful softwares such as photoshop.

The process can be further improved with the help of the application of different algorithms for the segmentation of the images.

\section{B. CONCLUSION AND FUTURE SCOPES}

In this paper a method for digital image forgery detection is proposed and is based on block based and key point feature based method. By integrating this method if one method fails to detect forgery then other method detects it and vice versa and the detection rate and efficiency will increase. For the detection features from the images are extracted. The input images were over segmented based on the SLIC algorithm. The features extracted were then employed for the matching of the blocks and the Local feature points identified were matched resulting in the identified similar portions in the images. The proposed method provides the better results than the existing system. The process can be further improved with the help of the application of different algorithms for the segmentation of the images. In the proposed approach the number of blocks of the images were calculated based on the input image.

\section{REFERENCES}

[1] Rafael C.Gonzalez.Richard E.Woods.(1992).Digital Image Processing $\left(3^{\text {rd }}\right.$ edition $)$

[2] Lockergnomer (2011) "Real World Application of Image Compression" Journal of information engineering and applications ISSNN 2224-5758

[3] Andrew B.Watson,NASA Ames Research,'Image Compression Using The Discrete Cosine Transform", Mathematica Journal

[4] M.Stumptl, Digital Watermarking”, University of Southampton, 2001.

[5] Nikolog N.ponomarenko Vladimir V,Lukin Karen Egizarian,Jaako Astola.DCT based High Quality Image Compression.

[6] Swastik Das and Rashi Ranjan Sethy ,"A thesis on image compression using Discrete Cosine Transform and Descrete Wavlet Transform ",Guided by :prof.R.Baliarsingh dept of computer science and engineering,national institute of Rourkela

[7] G. Li, Q. Wu, D. Tu, and S. Sun, "A sorted neighborhood approach for detecting duplicated regions in image forgeries based on DWT and SVD," in Multimedia and Expo, 2007 IEEE International Conference on, 2007, pp. 1750-1753.

[8] weiqiLuo, Jiwu Wang, GuopingQiu "Robust detection of Regionduplication Forgery in Digital Image" The 18th International Conference on Pattern Recognition (ICPR'06) 0-7695-2521-0/06 $\$ 20.00$ (C) 2006.

[9] 3.X. Bo, W. Junwen, L. Guangjie, and D. Yuewei, "Image copymove forgerydetection based on SURF," in Multimedia Information Networking and Security (MINES), 2010 International Conference on, 2010, pp. 889-892.

[10] 4.Vincent Christlein, Student Member, IEEE, Christian Riess, Associate Member, IEEE, Johannes Jordan, Student Member, IEEE, CorinnaRiess, and Elli Angelopoulou, Member, IEEE
[11] 5.R. Achanta, A. Shaji, K. Smith, A. Lucchi, P. Fua, and S. Susstrunk, "SLIC superpixels compared to state-of-the-art superpixel methods," IEEE Trans Pattern Anal Mach Intell, vol. 34, pp. 2274-82, Nov 2012.

[12] Sergio Bravo-Solorio and Asoke K. Nandi " Automated detection and localisation of duplicated regions affected by reflection, rotation and scaling in image forensics"Department of Electrical and Engineering and Electronics,The University of Liverpool,BrownlowHill,Liverpool L69 3Gj,UK 2011.

[13] Karen Less "Image compression using wavelets", Report of M.S.Zooz.

[14] Anil Kumar Katharotiya, Swati Patel, Mahesh Goyani department computer engineering, LDCE, GTU, comparative analysis between DCT and DWT tech of image processing.

[15] Nishit Gupta, Anant Kumar, Abhishek Mishra, Mayank Kumar, International journal of computer science and mobile computing.

[16] M.tech research scholar, Asso. Proff. And HOD, A survey on the digital image copy move forgery detection technique.

[17] Barnali Sharma, Gypsi Nandi, International journal of advanced research in computer science and software engineering.

[18] M.Ali Qureshi, M.Deriche a review on copy move image forgery detection technique.

[19] Radhakrishna Achanta, Appu Shaji, Kevin Smith, Aurelien Lucchi,Pascal Fua, and Sabine S $\square$ usstrunk School of Computer and Communication Sciences (IC) Ecole Polytechnique F_edrale de Lausanne (EPFL). 